\title{
Virtual Network Embedding in Elastic Optical Data Center Network
}

\author{
Aidong Su ${ }^{1, a^{*}}$ and Yongyi Zhang ${ }^{1, b}$ \\ ${ }^{1}$ Dalian Airforce Communication NCO Academy, P. R. China \\ asuaidong@126.com, ${ }^{\mathrm{b}} 80834567 @ q q . c o m$
}

Keywords: VON embedding; cloud computing; elastic optical network; data center

Abstract. The booming internet services urge the research on the cloud computing and the network resource utilization. The combination of elastic optical network and data center can solve the network resources deficient problem and the computing resources unbalanced problem. Virtual optical network embedding provides the way of resource high-efficiency. In this paper, we propose a novel virtual networks embedding algorithm orienting spectrum resource, and the simulation results verify its superiority on increasing spectrum resource utilization and reducing demand blocking rate.

\section{Introduction}

With the development of Internet, bandwidth demand is booming. The construction of flexible and large capacity optical network becomes important [1]. Wavelength Division Multiplexing (WDM) optical network, allocating network resource in a one-size-fits-all manner, leads to inefficient resource utilization and low flexibility. By introducing the orthogonal frequency division multiplexing (OFDM) and breaking through the fixed bandwidth spacing restriction between wavelength tunnels, elastic optical network utilizes spectrum resources efficiently. Meanwhile, with the widespread use of cloud computing and virtualization technology in data centers (DC), the virtual network embedding (VNE) becomes a challenge in the cloud data DC networks (DCNs) [2-5]. It enables the co-existence of multiple virtual networks on the same substrate network by sharing the available resources. Thus, VNE in elastic optical DCNs is widespreadly concerned.

This paper first describes VNE problem in elastic optical DCNs and presents the corresponding mathematical model. For static traffic, we design a virtual network embedding algorithm based on the layered auxiliary graph referred to as VNE orienting spectrum resource (VNE-OSR). The proposed algorithm can integrate four different service ordering strategies.

Simulation results show that, in terms of improving network resource utilization and reducing the blocking rate, the proposed VNE-OSR algorithm reflects good performances.

\section{Elastic Optical Data Center Network Virtualization}

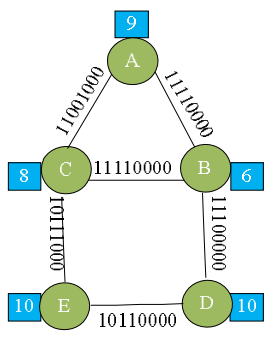

(a) Substrate networks

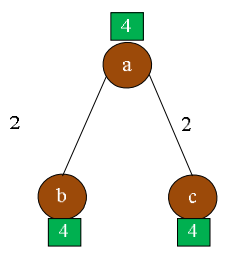

(b) VON demand

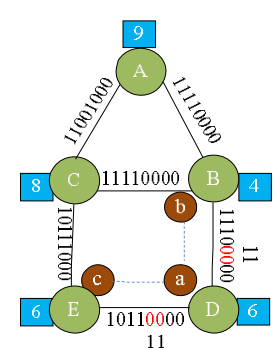

(c) VON Embedding result

Fig. 1 Schematic diagram of virtual network embedding

Optical DCN virtualization equates the combination of the virtual node embedding and the virtual link embedding, i.e., the mapping from virtual optical network (VON) to physical networks [6]. That includes 1) selecting appropriate servers (or DC) for the computing resource requests of virtual nodes, i.e., the mapping from virtual nodes to substrate computing elements, and 2) allocating appropriate fiber links and spectrum for virtual links, i.e. the mapping from virtual links to fiber links [7]. 
Concretely, as shown in Fig. 1(a), there are 5 servers and 6 fiber links in the substrate networks. There exist 8 spectrum slots in each fiber link, which can be expressed by an eight-binary-array, where "1" denotes this spectrum slot has been occupied; otherwise, it's " 0 ". The number besides each server (or DC) indicates the remainder computing resource. As shown in Fig. 1(b), the arriving VON needs 3 virtual nodes of 4 computing resources and 2 virtual links of 2 continuous slots. Fig. 1(c) shows the result of VON embedding, i.e., the virtual nodes $a, b$ and $c$ are mapped accordingly to servers $D, B$ and $E$, and the virtual links $a \leftrightarrow b$ and $a \leftrightarrow c$ are mapped accordingly to $D \leftrightarrow B$ and $D \leftrightarrow E$. The VNE in the elastic optical DCNs can neatly distribute spectrums according to demands, so it can rise the spectrum resource utilization, and meanwhile, VNE mainly orients the scene where the DCN power-system fails and then recovers gradually. In this situation, there exist many improper servers since a power failure and scarce server-computing-resource will lead to many blocked VON demands, thus it is very valuable to research.

\section{Problem Description}

The elastic optical DCNs have the abstracted substrate topology $G^{s}\left(V^{s}, E^{s}\right)$, where $V^{s}$ represents the set of substrate nodes, and $E^{s}$ represents the set of bi-directional links (each link is consist of two reversed-unidirectional fibers). Each substrate node $n \in V^{s}$ has a certain amount of available computing resource $c_{n}$. The spectrum resource in each fiber link $e \in E^{s}$ is divided into spectrum slots with the same bandwidth, and each spectrum slot corresponds with an OFDM sub-carrier, i.e. each fiber link constitutes a series of continuous sub-carriers. This situation could be expressed by a binary array $b_{e}$ with $B$ elements, where $B$ represents the maximum sub-carrier quantity in each fiber.

Each VON request could be indicated by non-directional graph $G^{r}\left(V^{r}, E^{r}\right)$, and each virtual node $j \in V^{r}$ has its computing resource request $m_{j}$. In the same VON, any bandwidth sub-request among all virtual links is equal, so the bandwidth request of each virtual link $k \in E^{r}$ is indicated by $n^{r}$, i.e., it is the continuous sub-carrier amount which need be assigned to the virtual link.

Each fiber link has the same quantity of sub-carriers, and as mentioned above, any required bandwidth in each virtual link in the same VON request has the coincident amount. The core of VON problem is to map a VON request into substrate networks, i.e. the mapping from virtual nodes into substrate nodes and the mapping from virtual links into the fiber links. For the static VON embedding problem, given that all the request demands were not blocked, the target of the VON embedding algorithm is minimizing the maximum sub-carrier serial number used in all fiber links.

\section{Virtual Network Embedding Algorithm}

We propose a novel VNE algorithm based on the layered auxiliary graph (LAG) referred to as VNE orienting spectrum resource (VNE-OSR) for static demands, and it takes two phases: the computing resource allocation for virtual nodes and the bandwidth resource allocation for virtual links. The algorithm can allocate appropriate spectrum resource according to the demand actual size.

VNE-OSR first tries to construct a LAG according to virtual link bandwidth requirements of a VON and the online-service bandwidth-condition of fiber links. If a LAG is built successfully, we execute the mapping of nodes and links on this graph; otherwise, we block the demand.

Table 1 shows the pseudo-code of VNE-OSR. Lines 2-7 express the process of constructing a LAG, and describe how to transport a VON demand mapping from substrate networks to a certain

LAG. The algorithm orderly checks each fiber whether $n^{r}$ available continuous spectrum slots exit. If there exist sufficient spectrum slots, we insert the fiber into the $\mathrm{LAG}_{i}$, where $i$ is the stating spectrum slot index. When all fibers are checked up, the algorithm will check interconnecting elements on $\mathrm{LAG}_{i}$, and forms some sub-graphs. And then it sorts these sub-graphs in the descending order based on the 
node number, where node $\left(G_{k}^{s u b}\right)$ denotes the node number in $G_{k}^{s u b} \cdot\left|V^{r}\right|$ denotes the virtual node number in a embedding request $V^{r}$. Lines 8-11 run the node mapping and the link mapping.

Table 1 Pseudo-code of VNE-OSR algorithm

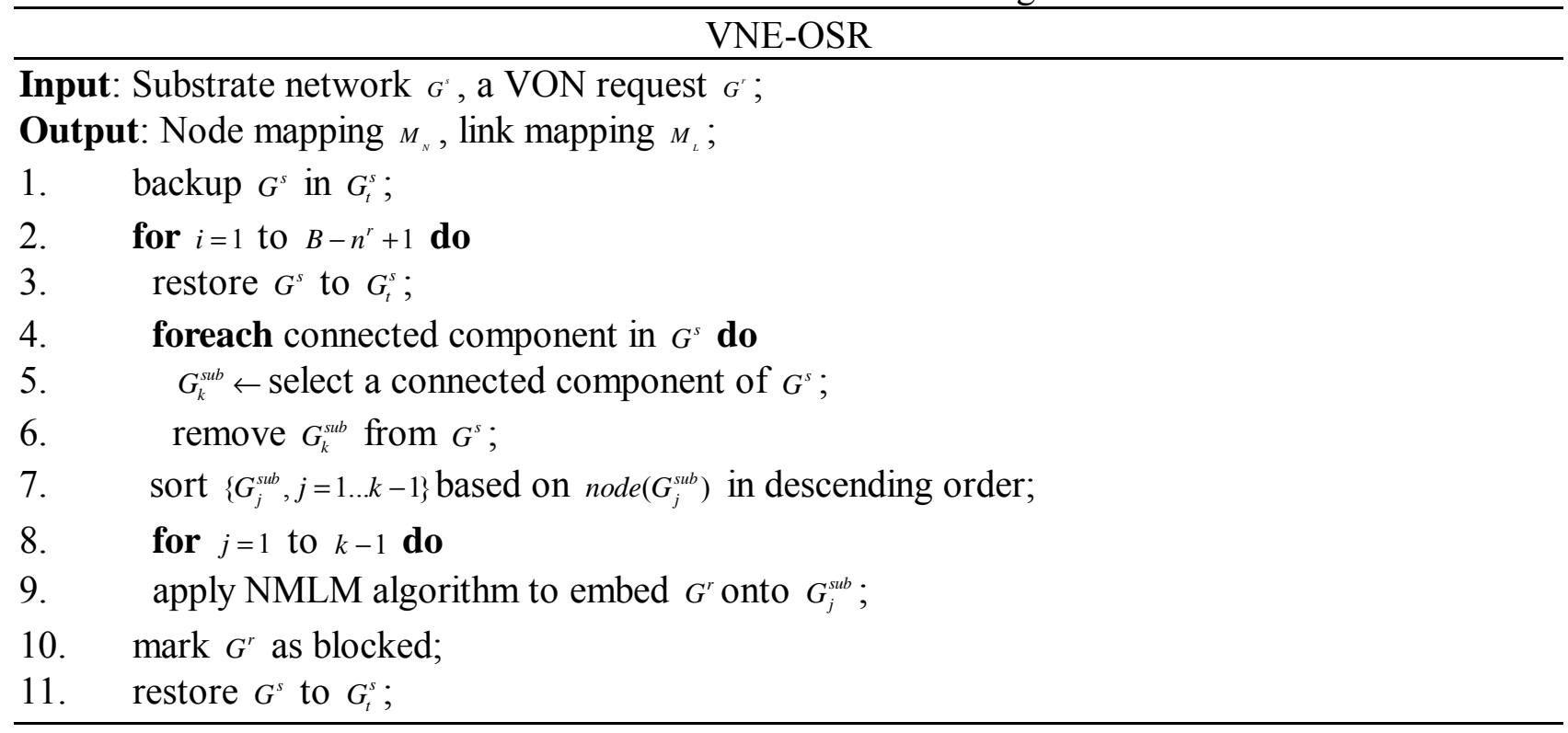

\section{Simulation}

Simulation Setting. We adopt NSFNET as the testing topology. Each fiber link consists of a pair of reversed-unidirectional fibers. The maximum sub-carrier serial number (MSSN) occupied in substrate networks and the mean blocking probability (MBP) are the test merits. MSSN is calculated by the equation (1), where $f_{s}$ is binary, and if the sub-carrier is occupied, $f_{s}=1$; or, $f_{s}=0$.

$$
M S S N=\max s \cdot f_{s} .
$$

Results and Analysis. Based on the different service sequence of demands, we combine the proposed VON embedding algorithm with four different ordering strategies, that is, first fit based VNE-OSR algorithm (VNE-FF), bandwidth fit based VNE-OSR algorithm (VNE-BF), computing fit based VNE-OSR algorithm (VNE-CF) and resource fit based VNE-OSR algorithm (VNE-RF). We do this simulation for two targets: 1) with efficient bandwidth resource, under the condition where the system can serve all demands, we compare the four algorithms by MSSNs in fiber links; 2) with limited bandwidth resource, we compare MBPs. All demands can be served and there are efficient computing and bandwidth resources. We suppose there are 300 sub-carriers in each fiber, and 300 computing resource capacity in each physical node (DC).

In Fig 2, in VONs, the bandwidth requirements of the virtual links range from 2 to 4, and the demand scope ranges from 10 to 80. With the increasing demands, the occupied MSSNs rise. MSSN of VNE-FF is the highest and it performs worst. Thus, for static demands, the demand service sequence can effect MSSNs. Compared with VNE-FF, other three algorithms perform better.

In Fig. 3, we suppose there 50 sub-carriers in each fiber and 800 computing resource capacity in each physical node. For VONs, the bandwidth requirements of virtual links range from 2 to 5 and the demand scope range from 20 to 200. When the demands are less than 60, all MBPs are 0 . With the increasing demand scope, all MBPs rise. That's because, under the limited bandwidth resource in fibers, the small demand scope leaves more reminder bandwidth resource, which can serve more demands and reduce MBP, and whereas the opposite. And then, as shown in Fig. 3, VNE-BF gains the highest MBP and performs worst, inversely, VNE-CF performs the best. That's because, VNE-BF 
follows a service order based on the bandwidth requirement sequence and it firstly serves the biggest bandwidth requirement demand, leading into the more occupied bandwidth resource in fibers. Thus, the rest resource can't serve all the subsequent demands.

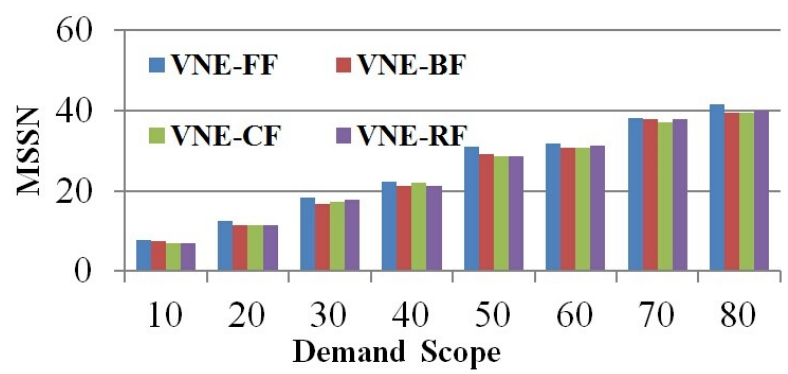

Fig. 2 Comparison of MSSNs with different demands among VNE-FF, VNE-BF, VNE-CF and VNE-RF

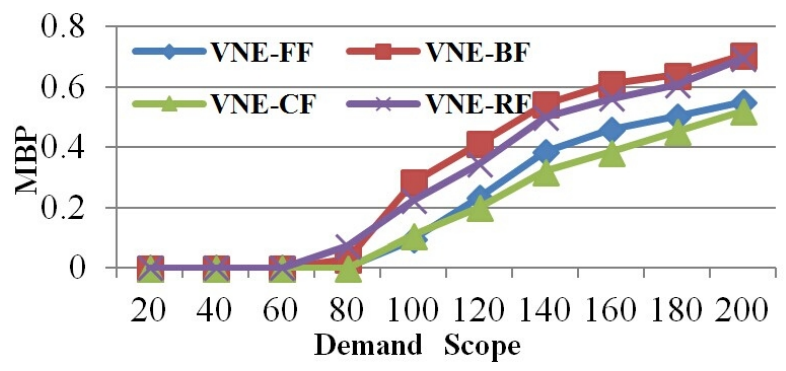

Fig. 3 Comparison of MBPs with different demands among VNE-FF, VNE-BF, VNE-CF and VNE-RF

\section{Conclusion}

It is valuable to research the virtual optical network embedding into data center networks or a single data center. This paper proposes a VNE algorithm orienting spectrum resource maximum utilization. The simulation results testify the advantage of our algorithm in the resource efficiency.

\section{References}

[1] S. Sakr, A. Liu, D. M. Batista, et al. "A Survey of Large Scale Data Management Approaches in Cloud Environments”, IEEE Communications Surveys \& Tutorials, 2011, 13(3): 311-336.

[2] C. Kachris, I. Tomkos. "A Survey on Optical Interconnects for Data Centres", IEEE Communications Surveys \& Tutorials, 2012, 14(4): 1021-1036.

[3] M. Jinno, H. Takara and B. Kozicki. "Concept and enabling technologies of spectrum-sliced elastic optical path network (SLICE)”, ACP, 2009, pp. 1-2.

[4] M. Jinno, H. Takara and B. Kozicki. "Spectrum-Efficient and Scalable Elastic Optical Path Network: Architecture, Benefits, and Enabling Technologies", IEEE Communications Magazine, 2009, 47(6): 66-73.

[5] M. Jinno, H. Takara and B. Kozicki. "Dynamic optical mesh networks: drivers, challenges and solutions for the future", ECOC, 2009, pp. 1-14.

[6] L. Gong, Z. Q. Zhu. "Virtual Optical Network Embedding (VONE) over Elastic Optical Networks", Journal of Lightwave Technology, 2014, 32(3): 450-460. 
[7] L. K. N. Georgakilas, A. Tzanakaki, M. Anastasopoulos, et al. "Converged Optical Network and Data Center Virtual Infrastructure Planning”, IEEE/OSA Journal of Optical Communications and Networking, 2012, 4(9): 681-691. 